\title{
A study of Er:YAG laser ablation using X-ray microtomography
}

Sequential 3D X-ray microtomographic measurement of enamel and dentine ablation by an Er:YAG laser

\section{Mercer, P. Anderson and G. Davies Br Dent J 2003; 194: 99-104}

\section{Objective}

To demonstrate the progression of crater growth during repeated sequential application of an Er:YAG laser to enamel and dentine, monitored using X-ray microtomography (XMT).

\section{Design}

A single centre study in which laser craters were created in blocks cut from human enamel and/or dentine under standardised and known conditions and then studied using XMT to obtain visualisation and quantification of the effects.

\section{Setting}

University setting, UK, 2001.

\section{Main outcome measures}

Success was judged by an ability to obtain useful 3D XMT reconstructions of the blocks during crater development, and to make measurements from these data. These measurements were compared with data obtained from similar studies using different measurement techniques.

\section{Results}

Time sequences of 2D and 3D images were obtained which demonstrated the progression of laser craters in enamel and dentine. Quantitative measurements from these data enabled values to be derived for the rate of progression of crater depth per unit energy, and the volume of hard tissue removed per unit energy. These values were compared with data derived from other studies and shown to be broadly comparable. However, the present study is unique in that these values were obtained from a series of measurements of the same craters over time.

\section{Conclusions}

3D X-ray microtomography is shown to be a useful tool for quantitative measurements in dental research. For the Er:YAG laser, the relationship of laser crater depth and volume of mineral removed to applied energy was found to be linear.

\section{IN BRIEF}

- X-ray microtomography is a useful 3D microscopic method in hard tissue dental research.

- X-ray microtomography images may be used for quantitative measurement.

- Results obtained using this method to measure laser (Er:YAG) ablation of dental hard tissues are compared with other studies.

\section{COMMENT}

In the four decades since the first experiments involving laser applications to teeth, a wealth of information on laser-tooth tissue interactions has been amassed and laser use in clinical dentistry is growing, including tissue ablation. This paper describes experiments using a non-destructive laboratory technique (X-ray microtomography - XMT) to characterise and quantify the tissue loss when using an Erbium:YAG laser, which is currently available for clinical use. The authors have developed an extremely sophisticated laboratory technique which enables them to precisely reposition their samples and thereby carry out accurate comparisons of sequential laser pulse effects on enamel and dentine, both quantitatively and qualitatively.

The production of 3D slice images has permitted the authors to carry out painstaking measurements of the laser craters after each pulse, effectively creating a quasi-real-time measurement tool for mineral loss. With its high resolution, this XMT technique will be of considerable value in a variety of laboratory (and in situ) studies investigating enamel and dentine mineral content, not only in relation to lasers but also other areas, notably in cariology.

If lasers are to be of widespread application as an ablative tool in clinical dentistry it is essential to determine their precise quantitative effects and this study has not only provided such information for one specific laser but also, in so doing, has demonstrated for the first time, a non-destructive laboratory technique capable of providing the data required to achieve this sequentially on the same sample.

In relation to laser pulse effects, the use of XMT in this study demonstrates that there is a linear relationship between laser crater depth development and applied energy in enamel and dentine. In addition, the study confirms that, with successive laser pulses, the diameter of the crater (1) does not enlarge significantly at the impact site and (2) diminishes as a function of depth.

Whilst there is a world of difference between the exacting scientific rigour in laboratory experiments using lasers at precisely fixed distances from tooth surfaces and the clinician working in a clinical environment, understanding the precise ablative effects of specific lasers will enable clinicians and researchers to determine the applicability of these lasers to particular clinical tasks. This careful study has added to the body of knowledge in this developing area and provided a useful tool to increase our understanding of the mechanisms involved.

C. Longbottom, Senior Lecturer in Preventive and Children's Dentistry, Dundee Dental School 